BULL. AUSTRAL. MATH. SOC.

VOL. $25(1982), 357-386$.

\title{
DIFFERENTIAL FORMS \\ WITH VALUES IN GROUPS
}

\author{
ANDERS KOCK
}

\begin{abstract}
In the context of synthetic differential geometry, we present a notion of differential form with values in a group object, typically a Lie group or the group of all diffeomorphisms of a manifold. Natural geometric examples of such forms and the role of their exterior differentiation is given. The main result is a comparison with the classical theory of lie algebra valued forms.
\end{abstract}

In synthetic differential geometry, one encounters formal manifolds, and in these it makes sense to talk about two points being neighbours [10]. In terms of this neighbour notion, it makes sense to talk about differential forms with values in a group $G$. This is equivalent to a classically considered notion of Lie algebra valued differential form (namely with values in the lie algebra of $G$ ), and the comparison between these two notions is the main result presented here. However, the definition of coboundary of $O-$ and 1 -forms with values in $G$ is, both from the analytic and geometric viewpoint, more natural than the classical Lie algebra valued notions. Thus, the Maurer-Cartan form $\Omega$ for a Lie group $G$ appears as the coboundary of the identity map of $G$ (which is a zero-form). In particular, it is closed, $d \Omega=0$, and this can be reinterpreted as the Maurer-Cartan formula.

Also, the two well known lemmas (cf., for example, [6] or [4])

Received 12 November 1981. This research was partially supported by the Australian Research Grants Committee. A preliminary report on some of the results is printed in Cahiers Topologie Géom. Différentielze 22 (1981), $141-148$. 
concerning maps from connected and simply connected manifolds into a lie group.become just the statements that $H^{0}(M, G)=G$ and $H^{l}(M, G)=0$, where $H$ denotes "deRham cohomology with values in $G$ ". The result about $H^{1}$ (which can be expressed: "closed 1 -forms are exact") is proved in $\S 7$. The crux of the comparison is that "our" $d \omega$ corresponds to the Lie algebra valued $d \bar{\omega}+\frac{1}{2}[\bar{\omega}, \bar{\omega}]$, where $\bar{\omega}$ corresponds to $\omega$.

The notion of $G$-valued form also works for the case where $G$ is a. "big" group, like $\operatorname{Diff}(F)$, for $F$ a (formal) manifold, and the "connection form" associated to a distribution (or connection) can be explained in straightforward geometric terms as a Diff( $F)$-valued form. We attempt this geometric explanation in $\$ 1$, where we also try to give a geometric explanation of "closed forms are exact", as being infinitesimally true by the very definition of coboundary of forms (for "linear" forms, a similar explanation was given in [13]; ef. [11]). However, the finite integration theory does not (and should not) work for big groups like $\operatorname{Diff}(F)$; this is commented on in $\S 8$.

I want to acknowledge several discussions with A. Joyal; first of all he has a for a long time advocated that one should define and utilize some notion of 1-neighbour in the synthetic context, and in particular consider the notion of infinitesimal simplex derived from it (cf. (1.1) and $\$ 3$ below). Secondly he, in collaboration with R. Bkouche, considered the dual cochain notion, with values in a commutative group; also they considered a connection notion, of which ours is a special case, namely: an action of the graph of the 1-neighbour relation for $M$ on an arbitrary object over $M$. They then defined its curvature to be a measurement of the defect of associativity of this action. For the special case we consider, the coboundary of the connection form is such a measure.

We remind the reader of the following notation and terminology from [10]: if $M$ is a formal manifold and $x \in M$, we have a subset $M_{1}(x)$ containing $x$, called the l-monad around $x$. It consists of the 1-neighbours of $x$. We use the word "neighbour" instead of 1-neighbour throughout the present article. The notation $x \sim y$ or $x-y$ means that $x$ and $y$ are neighbours (this is a symetric relation). Likewise $M_{\infty}(x)$ denotes the ${ }^{\infty}$-monad around $x ;$ it is $\cong D_{\infty}(n)$ (that is, $\infty$-monad 
around $0 \in R^{n}$ ) for some $n$, and is an étale subset.

Also, since we shall compare our group-valued forms with more classical vector space valued "multilinear alternating" forms, we shall consistently call the latter linear forms. For an account of such in synthetic differential geometry, we refer the reader to [11] or [13]. Linear forms, and the coboundary operator for them, are consistently denoted with an "overbar": $\bar{\alpha} \bar{\omega}$ and so on.

\section{Geometric meaning}

A (number valued) 1 -form is a law $\omega$ which to a pair of neighbour points $x, y$ associates a number $\omega(x, y)$, for example the amount of work required to go from $x$ to $y$; we require $\omega(x, x)=0$. We may also have 1 -forms with values in transformation groups - for instance going from $x$ to a neighbour point $y$ on a space curve effects a change (rotation) of the Frenet frame; if $x=y$, the rotation is the identity.

If we "transfinitely often" keep passing from a point to a neighbour point, thus tracing out a curve, the infinitesimal "amounts of work" or "changes" $\omega(x, y)$ will accumulate to a finite number, or change, respectively. This is the process of (curve-) integration which gives us finite numbers, or finite transformations, respectively.

If we go along different curves or paths from one point to another, we may not get the same value by the integration, not even if the two curves are homotopic. Alternatively, going around a closed curve may not give us the neutral element $e$ of the group as value, not even if the curve is null-homotopic. However, in the latter case we would expect that if the value obtained by curve integration along any infinitesimal closed curve is $e$, then we also get $e$ by going around finite null-homotopic curves.

We want to find conditions under which this reasoning is correct. First of all, what are the infinitesimal closed curves by which we fill in the finite null-homotopic ones? We take these to be triangles $x, y, z$, where $(x, y),(y, z)$ and $(z, x)$ are neighbours: 


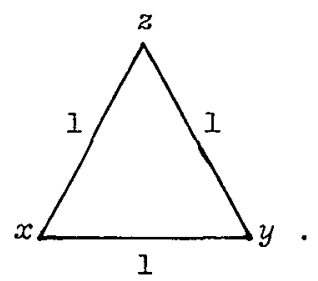

We define the curve integral around this (starting in $x$ ) to be

$$
\omega(z, x) \cdot \omega(y, z) \cdot \omega(x, y),
$$

where - denotes the multiplication of the value group. (We choose the ordering in (1.2) rather than the more forward-looking

$$
\omega(x, y) \cdot \omega(y, z) \cdot \omega(z, x)
$$

because we want the value groups to be transformation groups which act from the left.) The element (1.2) is denoted $d \omega(x, y, z)$. In fact, $d w$ is a 2 -form with values in the group in question, and its vanishing (that is, having $e$ as its only value) means precisely that integrating $\omega$ around any triangle (1.1) gives $e$.

If we can perform the passage from infinitesimal to finite (nullhomotopic) closed curves, alluded to above, we have

$$
\text { (1.3) } d \omega=e \Rightarrow\left(\int_{k} \omega \text { depends only on the end points of } k\right)
$$

(in simply connected domains $M$ ) and by the standard procedure, this in turn leads to the construction of a function $f: M \rightarrow G$ such that

$$
f(y) \cdot f(x)^{-1}=\omega(x, y) \text { for } x \sim y
$$

namely letting $f(z)=\int_{k(a, z)} \omega$, where $a$ is a point chosen once and for all, and $k(a, z)$ is any curve starting in $a$ and ending in $z$. How (1.4) can be expressed $d f=\omega$, so that (1.3) says

$$
d \omega=0 \Rightarrow \omega=d f .
$$

As an example of how one may arrive at a l-form with values in the group $\operatorname{Diff}(F)$ of all bijective maps $F \rightarrow F$, consider a distribution $D$ on $I A \times F$ transverse to the fibers of the projection to $M$, where $M$ and $F$ are formal manifolds; this means that around each $(x, u) \in M \times F$, 
there is given a subset $D(x, u) \subseteq M_{I}(x, u)$ which by proj: $M \times F \rightarrow M$ maps bijectively to $M_{1}(x)$. Thus, if $u \in F$, and $x \sim y$ in $M$, there is a unique $u^{\prime}$ with $\left(y, u^{\prime}\right) \in M(x, u)$. Thus, the pair $x, y$ defines an automorphism of $F, u \mapsto u^{\prime}$, which we denote $\omega(x, y)$. Thus $\omega$ is a l-form on $M$ with values in $\operatorname{Diff}(F)$ (which is a very big group - not a formal manifold).

Heuristically, the "line" connecting $x$ to $y$ lifts (for the given $u$ ) to the "line" connecting $(x, u)$ to $\left(y, u^{\prime}\right)$, and this "line" lies in $D(x, u)$, and thus $D$ defines an infinitesimal path-lifting. The finite curve integration of $\omega$ amounts to lifting of finite paths. To say $d \omega(x, y, z)=e$, where $(x, y, z)$ is an infinitesimal triangle (I.I) in $M$, means that (for any initial value $u \in F$ ), the triangle lifts to a closed triangle ("no infinitesimal holonomy"). To say $\omega=d f$ for some $f: M \rightarrow \operatorname{Diff}(F)$ (locally) can by a little combinatorics be seen to imply that $D$ arises from a foliation, the leaf through $(x, u)$ being the graph

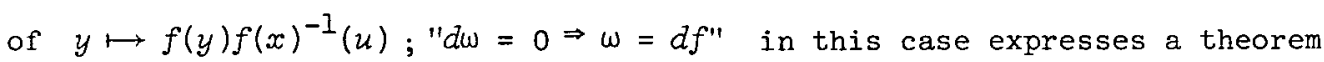
about integrability of distributions. In $\S 8$ we will demonstrate that the condition $d \omega=0$ "is" the usual analytic condition in Frobenius' Theorem about distributions.

Now, Diff $(F)$ does not "admit integration over finite intervals" in the sense to be explained in $\$ 6$, whence we cannot really derive (*), unless we can assert that the form in question takes values in a subgroup of $\operatorname{Diff}(F)$ that does admit integration, or alternatively, if $M$ is small enough. We discuss this in $\$ 8$.

\section{Some infinitesimal arithmetic}

We assume $R$ to be of line type in the strong sense of [9], or satisfy Axiom $1^{W}$ of [11].

As usual, we define

$$
D(n)=\left\{\left(d_{1}, \ldots, d_{n}\right) \in R^{n} \mid d_{i} \cdot d_{j}=0 \forall i, j\right\} \subseteq R^{n} .
$$

Note that $D(2 n) \subseteq D(n) \times D(n)$. An intermediate object is 


$$
\begin{aligned}
& \tilde{D}(2 n)=\left\{\left(d_{1}, \ldots, d_{n}\right),\left(\delta_{1}, \ldots, \delta_{n}\right) \mid\left(d_{i} \cdot \delta_{j}+d_{j} \cdot \delta_{i}=0\right\}\right. \\
&\left.\wedge\left(d_{i} \cdot d_{j}=0\right) \wedge\left(\delta_{i} \cdot \delta_{j}=0\right) \forall i, j\right\} .
\end{aligned}
$$

We write $\mathrm{d}$ for $\left(d_{1}, \ldots, d_{n}\right)$. Note $M_{1}(v)=v+D(n)$ for $v \in R^{n}$. Note also $0 \sim \mathrm{d} \sim \delta \sim 0$ if and only if $(\mathrm{d}, \delta) \in \tilde{D}(2 n)$. Since 2 is invertible in $R$, we have furthermore $d_{i} \cdot \delta_{i}=0$ for any $(\mathrm{d}, \delta) \in D(2 n)$ and $i=1, \ldots, n$. We have

PROPOSITION 2.1. Any map $D(n) \rightarrow R^{m}$ with $0 \mapsto 0$ is the restriction of a unique Zinear map $R^{n} \rightarrow R^{m}$.

(For $n=1$, this is the basic ("Kock-Lawvere") axiom.)

PROPOSITION 2.2. Any map $D(n) \times D(n) \rightarrow R^{m}$ with $(0, \mathrm{~d}) \mapsto 0$ and $(\mathrm{d}, 0) \mapsto 0$, for alz $\mathrm{d} \in D(n)$, is the restriction of a unique bilinear $\operatorname{map} R^{n} \times R^{n} \rightarrow R^{m}$.

PROPOSITION 2.3. Any map $f: \tilde{D}(2 n) \rightarrow R^{m}$ with $(0, \mathrm{~d}) \mapsto 0$ and $(\mathrm{d}, 0) \mapsto 0$, for all $\mathrm{d} \in D(n)$, is the restriction of a unique bilinear skew symmetric map $R^{n} \times R^{n}+R^{m}$.

We give the proof for the last one only. It suffices to consider the case $m=1$. The Weil algebra defining $\tilde{D}(2 n)$ is

$$
k\left[x_{1}, \ldots, X_{n}, Y_{1}, \ldots, y_{n}\right] / I
$$

where $k$ is the ground field (assumed to be of characteristic not equal to $2)$, and $I$ is the ideal generated by all $X_{i} \cdot X_{j}, Y_{i} \cdot y_{j}$, and $X_{i} \cdot Y_{j}+X_{j} \cdot Y_{i} \cdot$ A $k$-linear basis for this Weil algebra is given by the classes (mod $I$ ) of the polynomials

$$
1, x_{1}, \ldots, x_{n}, y_{1}, \ldots, y_{n},\left(x_{i} \cdot y_{j}-x_{j} \cdot y_{i}\right)_{i<j} \text {, }
$$

as can easily be seen by recalling that a bilinear form is uniquely the sum of a symmetric and a skew one; and the $X_{i} \cdot y_{j}-X_{j} \cdot y_{i}$ for $i<j$ form a $k$-basis for the skew bilinear forms.

From Axiom $I_{k}^{W}$ it then follows (cf. [11], p. 92) that for any 
$f: \tilde{D}(2 n) \rightarrow R$, there are unique $r, s_{i}, s_{i}^{\prime}$, and $t_{i j}$ such that, for all $(d, \delta) \in \tilde{D}(2 n)$,

$f\left(\left(d_{1}, \ldots, d_{n}\right),\left(\delta_{1}, \ldots, \delta_{n}\right)\right)$

$$
=r+\sum s_{i} d_{i}+\sum s_{i}^{\prime} \delta_{i}+\sum_{i<j} t_{i j} \cdot\left(d_{i} \delta_{j}-d_{j} \delta_{i}\right) .
$$

If $f$ further satisfies the conditions of the proposition, the $r, s_{i}$, and $s_{i}^{\prime}$ are all 0 , and the $t_{i j}$ 's define the required bilinear skew map $R^{n} \times R^{n} \rightarrow R$. The uniqueness follows from the uniqueness of the $t_{i j}$ 's .

Note that a symmetric bilinear map $R^{n} \times R^{n} \rightarrow R$ has zero restriction to $\tilde{D}(2 n)$; and in particular:

PROPOSITION 2.4. For any bilinear $\phi: R^{n} \times R^{n} \rightarrow R$, we have

$$
\phi(d, \delta)=\frac{1}{2}(\phi(d, \delta)-\phi(\delta, d)) \text { for } a Z z(d, \delta) \in \tilde{D}(2 n) \text {. }
$$

\section{Group valued forms}

Let $M$ be a formal manifold. An infinitesimal $k$-simplex (Joyal) is a $(k+1)$-tuple of elements in $M,\left(x_{0}, \ldots, x_{k}\right)$ so that $x_{i} \sim x_{j}$, for all $i, j$. A $k$-form with values in a group $G$ is a law which to any infinitesimal $k$-simplex associates an element in $G$, and associates $e$ if two of the vertices $x_{i}$ and $x_{j}$ of the simplex are equal.

Such forms have, in another context, been considered by Bkouche and Joyal who use them (with $G=R$ ) to define deRham cohomology (unpublished). We shall be interested mainly in non-commutative groups $G$, and therefore only in low dimensions $k=0,1,2$.

A 0 -form on $M$ with values in $G$ is just a map $f: M \rightarrow G$. Its coboundary, $d f$, is the 1-form given by

$$
d f(x, y)=f(y) \cdot f(x)^{-1} \text { for } x \sim y .
$$

If $\omega$ is a 1 -form, we define

$$
d \omega(x, y, z)=\omega(z, x) \cdot \omega(y, z) \cdot \omega(x, y)
$$

for $x \sim y \sim z \sim x$; under suitable assumptions on $G$, dw will be a 
2-form on $M$, ef. Proposition 4.1. We denote (for $k=0,1,2$ ) by 0 the $k$-form whose only value is $e$. Clearly $d(d f)=0$. Since any map $g: M \rightarrow M^{\prime}$ between formal manifolds preserves the property of being neighbours:

$$
x \sim y \Rightarrow g(x) \sim g(y),
$$

a $k$-form $\omega$ on $M^{\prime}$ immediately gives rise to a $k$-form $g^{*}(\omega)$ on $M$. Clearly, $g^{*}(d \omega)=d\left(g^{*} \omega\right)$.

If the value group $G$ itself is a formal manifold, we have the identity map $i: G \rightarrow G$ as a 0 -form on $G$. Its coboundary $d i$ is the Maurer-Cartan form $\Omega$ on $G$ :

$$
\Omega(x, y)=d i(x, y)=y \cdot x^{-1} \text { for } x \sim y \text {. }
$$

Clearly

$$
d \Omega=0
$$

we shall see in $\$ 5$ that this is really the Maurer-Cartan formula. Also, for any $f: M \rightarrow G$,

$$
d f=d\left(f^{*} i\right)=f^{*}(d i)=f^{*} \Omega .
$$

\section{Certain infinitesimal curve integrals}

If $\omega$ is a $G$-valued 1 -form on a formal manifold $M$, we want to considex

$$
\omega(y, x) \cdot \omega(x, y) \text { for } x \sim y
$$

and

$$
\omega(z, x) \cdot \omega(y, z) \cdot \omega(x, y) \text { for } x \sim y \sim z \sim x .
$$

If $M=R^{n}$ (or any etale subobject thereof), these can also be written

$$
\omega(x+\mathrm{d}, x) \cdot \omega(x, x+\mathrm{d}) \text { for } \mathrm{d} \in D(n)
$$

and

(4.4) $\omega(x+\delta, x) \cdot \omega(x+d, x+\delta) \cdot \omega(x, x+d)$ for $(d, \delta) \in \tilde{D}(2 n)$.

These are to be thought of as infinitesimal "curve integral back and forth" and infinitesimal "curve integral around a triangle", respectively. 
We also want to consider the infinitesimal curve integral around a parallelogram:

(4.5) $\omega(x+\delta, x) \cdot \omega(x+d+\delta, x+\delta) \cdot \omega(x+d, x+d+\delta) \cdot \omega(x, x+d)$

$$
\text { for }(\mathrm{d}, \delta) \in D(n) \times D(n) \text {. }
$$

PROPOSITION 4.1. Let $G$ be a subgroup of the group Diff(F) of all bijective maps $F \rightarrow F$, where $F$ is a formal manifold. If $\omega$ is a 1-form with values in such $G$, we have:

(i) the value of (4.1) is $e$, for alz $x \sim y$, (expressing that $w$ is alternating); and

(ii) (4.4) gives $e$, for all $(\alpha, \delta) \in \tilde{D}(n)$, if and only if (4.5) gives $e$ for all $(\mathrm{d}, \delta) \in D(n) \times D(n)$.

Proof. For all of the five expressions, we consider their effect on a fixed $u_{0} \in F$. We choose a frame around $u_{0}$ and since the question is local, we may as well assume $F \subseteq R^{k}$ (étale subset); for (4.1) and (4.2) we also choose a frame around $x$, reducing them to (4.3) and (4.4) respectively; in particular, we assume $M \subset R^{n}$ (étale subset).

For fixed $y \in M, u \in F$,

$$
\omega(y,-)(u) \text { is a map } M_{1}(y) \rightarrow M_{1}(u),
$$

thus, by Proposition 2.1, given by a unique affine $R^{n} \rightarrow R^{k}$ whose linear part we denote $A(y, u,-)$, in other words

$$
\omega(x, x+\mathrm{d})(u)=u+A(x, u, \mathrm{~d}) .
$$

Let us note that

$$
\begin{aligned}
\omega(x+\mathrm{d}, x)(u) & =u+A(x+\mathrm{d}, u,-d) \\
& =u+A(x, u,-d)+D_{1} A(x, u,-d)(d),
\end{aligned}
$$

where $D_{1} A$ denotes partial Jacobian of $A$ with respect to its first variable. Since $D_{1} A(x, u, s)(t)$ is bilinear in $s$ and $t$, and $d \in D(n)$, the last term vanishes, so that we have

$$
\omega(x+d, x)(u)=u+A(x, u,-d)=u-A(x, u, d) .
$$

Now let us calculate the effect of (4.3) on $u$ : 


$$
\begin{aligned}
\omega(x+\mathrm{d}, x) \cdot \omega(x, x+\mathrm{d}) & (u) \\
& =\omega(x+\mathrm{d}, x)(u+A(x, u, d)) \\
& =u+A(x, u, \mathrm{~d})-A(x, u+A(x, u, d), \mathrm{d}) \\
& =u+A(x, u, \mathrm{~d})-A(x, u, d)-D_{2} A(x, u, d) A(x, u, \mathrm{~d})
\end{aligned}
$$

(where $D_{2} A$ is partial Jacobian with respect to the second variable). The last term here equals 0 because it again is from $B(d, d)$ for some bilinear $B$. So the effect of (4.3) on $u$ is $u$. This proves (i).

To prove (ii), we calculate (4.4) and (4.5) by the same technique; we get, after cancelling all $B(d, d)$ and $B(\delta, \delta)$ for $B$ bilinear, that

$$
(4.4)-u=D_{1} A(x, u, \delta)(\mathrm{d})+D_{2}(x, u, \delta)(A(x, u, d)), \quad(\mathrm{d}, \delta) \in \tilde{D}(2 n) .
$$

Similarly, we get

$$
\begin{aligned}
(4.5)-u= & D_{1} A(x, u, \delta)(\mathrm{d})-D_{1} A(x, u, d)(\delta) \\
& +D_{2} A(x, u, \delta)(A(x, u, d))-D_{2} A(x, u, d)(A(x, u, \delta))
\end{aligned}
$$

for $(d, \delta) \in D(n) \times D(n)$. The expression derived here is bilinear in $\mathrm{d}, \delta$; denote it $C_{x, u}(\mathrm{~d}, \delta)$. Thus $C_{x, u}: R^{n} \times R^{n} \rightarrow R^{k}$ is given by the same formula; it is clearly skew symmetric. Comparing it with the expression derived for (4.4), and using Proposition 2.4, we thus have

(4.7) $\left\{\begin{array}{l}(4.4)-u=\frac{1}{2} C_{x, u}(d, \delta) \text { for all }(d, \delta) \in \tilde{D}(2 n), \\ (4.5)-u=c_{x, u}(d, \delta) \text { for all }(d, \delta) \in D(n) \times D(n),\end{array}\right.$

from which (ii) follows, using Proposition 2.3.

\section{Lie algebra valued forms}

Our theory is, when it comes to coordinate calculations, equivalent to the classical theory of Lie algebra valued differential forms. To make this comparison, we first state two, generally useful, propositions.

PROPOSITION 5.1. Let $M$ and $N$ be formal manifolds. There is, for arbitrary $x \in M, u \in N$, a natural bijective correspondence between

(1) maps $M_{1}(x) \rightarrow N$ taking $x$ to $u$,

(2) Zinear maps $T_{x} M \rightarrow T_{u} N$, 
the passage from (1) to (2) given by sending $\varphi: M_{1}(x) \rightarrow N$ into $[t \mapsto \varphi$ ○ $\quad t]$.

This is well-known; see, for example, [10], Remark 6.1.

PROPOSITION 5.2. Let $M$ and $F$ be formal manifolds. There is, for arbitrary $x \in M$, a natural bijective correspondence between

(I) maps $M_{1}(x) \rightarrow \operatorname{Diff}(F)$ taking $x$ to id,

(2) Zinear maps $T_{x} M \rightarrow \operatorname{Vect}(F) \quad\left(\right.$ that is, $\left.T_{\text {id }}(\operatorname{Diff}(F))\right)$,

where $\operatorname{Vect}(F)$ is the vector space of all vector fields on $F$. The passage from (1) to (2) is given by sending $\varphi: M_{1} \rightarrow \operatorname{Diff}(F)$ to $\Phi: T_{x} M \rightarrow \operatorname{vect}(F)$, where

$$
\Phi(t)(u, d)=\varphi(t(d))(u), \quad t \in T_{x} M, \quad d \in D, \quad u \in F
$$

Proof. First, we argue that the $\Phi$ constructed is $R$-linear. It is easily seen to be homogeneous (that is commute with multiplication by scalars from $R$ ). But because $F$ is infinitesimally linear, $V=\operatorname{vect}(F)$ is an $R$-module for which Proposition I.10.2 of [11] can be applied, to give the linearity. We next produce the passage from (2) to (1). We choose a frame around $x \in M$, identifying $M_{1}(x)$ with $D(n) \quad(n=\operatorname{dim} M)$, and ${ }^{T} x^{M}$ with $R^{n}$. Let there be given a linear

$$
T_{x} M=R^{n} \stackrel{\Phi}{\longrightarrow} \operatorname{vect}(F)
$$

Let $\Phi_{i}=\Phi\left(\mathrm{e}_{i}\right)$ where $\mathrm{e}_{i}$ is the $i$ th canonical basis vector of $R^{n}$; $\Phi_{i}$ is a vector field on $F$. We define $\varphi: D(n) \rightarrow \operatorname{Diff}(F)$ by putting (for $u \in F$, and $\left.\mathrm{d}=\left(d_{1}, \ldots, d_{n}\right) \in D(n)\right)$

$$
\varphi(d, u)=\Phi_{1}\left(d_{1}, \Phi_{2}\left(d_{2}, \ldots, \Phi_{n}\left(d_{n}, u\right)\right) \ldots\right),
$$

where $\Phi_{i}$ is identified with a map $D \times F \rightarrow F$.

To see that the processes are mutually inverse, first note that the process $\varphi \mapsto \Phi$ described in the proposition in coordinates can be described as follows; it suffices to describe $\Phi_{i}$ : 


$$
\Phi_{i}(d, u):=\varphi(0, \ldots, d, \ldots, 0)(u)
$$

( $d$ in the ith place). To prove $\varphi \rightarrow \Phi \rightarrow \varphi$ leads back to the original $\varphi$ then means proving

$$
\begin{aligned}
\varphi\left(d_{1}, 0, \ldots, 0\right) \circ \varphi\left(0, d_{2}, \ldots, 0\right) \circ \ldots \circ \varphi\left(0,0, \ldots, d_{n}\right)(u) & =\varphi\left(d_{1}, \ldots, d_{n}\right)(u) .
\end{aligned}
$$

Both sides here (for fixed $u$ ) are maps $D(n) \rightarrow F$, and to see that they are equal, it suffices to see, by infinitesimal linearity of $F$ (which is a consequence of $F$ being a formal manifold), that they have equal restrictions to the $n$ "axes" of $D(n)$, which is evident from $\varphi(0, \ldots, 0)=$ identity .

Conversely, start with $\Phi$. It suffices to see that the old $\Phi_{i}\left(=\Phi\left(\mathbf{e}_{i}\right)\right)$ and the new $\Phi_{i}$ agree, that is to prove

$$
\Phi_{i}(d, u)=\varphi(0, \ldots, d, \ldots, 0)(u)
$$

but

$$
\varphi(0, \ldots, d, \ldots, 0)(u)=\Phi_{1}\left(0, \Phi_{2}\left(0, \ldots, \Phi_{i}\left(d, \ldots\left(\Phi_{n}(0, u)\right) \ldots\right)\right)\right),
$$

and the result follows because $\Phi_{k}(0, u)=u$.

If in Proposition 5.1 we take $N=G$ (a group which is a formal manifold) and $u=e$, the proposition expresses that we have a natural one-to-one correspondence between 1 -forms $\omega$ on $M$ with values in $G$, and "ordinary" linear differential l-forms $\bar{\omega}$ on $M$ with values in $T_{e} G(=L G)$ (for the "ordinary" notion of linear differential forms in synthetic setting, see [13]). Similarly, from Proposition 5.2 it follows that we have a natural one-to-one correspondence between l-forms $\omega$ on $M$ with values in $\operatorname{Diff}(F)$, and linear l-forms $\bar{\omega}$ on $M$ with values in the vector space $\operatorname{vect}(F)$ (which, even though it is not finite dimensional, admits synthetic calculus since each individual $T_{u} F$ does). Linear differential forms with values in $\operatorname{Vect}(F)$ have been considered classically; cf., for example, Haefliger, [5].

To complete the comparison, we also need to consider 2-forms.

PROPOSITION 5.3. Let $M$ be a formal manifold, and let $N$ be a 
formal manifold, or $\operatorname{Diff}(F)$, where $F$ is a formal manifold. Let $x \in M$ be arbitrary, and let $u \in N$ be arbitrary in the former case, and equal to the identity map in the latter. Then there is a bijective natural correspondence between

(1) those maps

$$
\tilde{M}_{2}(x)=\{(y, z) \mid x \sim y \wedge y \sim z \wedge z \sim x\} \stackrel{\varphi}{\longrightarrow} N
$$

which take $(x, z)$ and $(y, x)$ to $u$, and

(2) bilinear skew symmetric maps

$$
T_{x} M \times T_{x} M \stackrel{\Phi}{\rightarrow} T_{u} N
$$

(the codomain is $\operatorname{Vect}(F)$, if $u=e \in \operatorname{Diff}(F)$ ).

Proof. Here we have to do the whole work in coordinates, by choosing frames, and then prove independence of the choice. Let us do the case $N=\operatorname{Diff}(F)$. So identify $\tilde{M}_{2}(x)$ with $\tilde{D}(2 n)$. For fixed $u \in F$ we have, by choosing a frame around $u$ and working with coordinates there,

$$
\varphi(\mathrm{d}, \delta)(u)=u+A_{u}(\mathrm{~d}, \delta),
$$

where $A: \tilde{D}(2 n) \rightarrow R^{k} \quad(k=\operatorname{dim} F)$ takes $(\mathrm{d}, 0)$ and $(0, \delta)$ to zero. Now $A$ extends uniquely to a skew bilinear $R^{n} \times R^{n} \rightarrow R^{k}$, by Proposition 2.3. Thus the information contained in $\varphi$ is: to each $u$, a skew bilinear $R^{n} \times R^{n} \rightarrow R^{k}$, which is also the information in (2). We omit the details about independence of choice of frames, and so on.

From Proposition 5.3 follows that we have a bijective correspondence between 2-forms $\theta$ on $M$ with values in $G$, and ordinary "linear" 2-forms $\bar{\theta}$ on $M$ with values in $L G$ for any $G$ which is either a formal manifold, or $\operatorname{Diff}(F)$ with $F$ a formal manifold.

If we let $\bar{a}$ denote exterior differentiation of ordinary linear differential forms, we may ask what is the relationship between $\overline{d \omega}$ and $d \bar{\omega}$ ? The answer involves the Lie bracket structure on $L G$ (calculated via right invariant vector fields on $G$ ).

THEOREM 5.4. Let $G$ be a group which is a formal manifold, or $\operatorname{Diff}(F)$ for $F$ a formal manifold. Let $M$ be a formal manifold, and $w$ 
a 1-form on $M$ with values in $G$. Then, for $w_{1}, w_{2} \in T_{x}^{M}$,

$$
(\overline{d \omega})\left(w_{1}, w_{2}\right)=\frac{2}{2}\left(\overline{d \omega}\left(w_{1}, w_{2}\right)+\left[\bar{\omega}\left(w_{1}\right), \bar{\omega}\left(w_{2}\right)\right]\right) .
$$

Equivalently

$$
(\overline{d \omega})=\frac{1}{2}\left(\bar{d} \bar{\omega}+\frac{2}{2}[\bar{\omega}, \bar{\omega}]\right),
$$

where

$$
[\bar{\omega}, \bar{\theta}](u, v):=[\bar{\omega}(u), \bar{\theta}(v)]-[\bar{\omega}(v), \bar{\theta}(u)] .
$$

Proof. Since the former case is easily reduced to the latter, we shall only do the $\operatorname{Diff}(F)$ case. We may do it in coordinates. We use notation as in $\$ 4$. For $(d, \delta) \in \tilde{D}(2 n)$, we calculated, in (4.7),

$$
d u(x, x+d, x+\delta)(u)=u+\frac{1}{2} C_{x, u}(d, \delta)
$$

with

$$
\text { (5.3) } \begin{aligned}
C_{x, u}\left(w_{1}, w_{2}\right) & =D_{1} A\left(x, u, w_{2}\right)\left(w_{1}\right)-D_{1} A\left(x, u, w_{1}\right)\left(w_{2}\right) \\
+ & D_{2} A\left(x, u, w_{2}\right)\left(A\left(x, u, w_{1}\right)\right)-D_{2} A\left(x, u, w_{1}\right)\left(A\left(x, u, w_{2}\right)\right)
\end{aligned}
$$

for all $\left(\mathrm{w}_{1}, \mathrm{w}_{2}\right) \in R^{n} \times R^{n}$. Thus the skew bilinear $R^{n} \times R^{n} \rightarrow R^{k}$ giving the coordinate information of $\overline{d \omega}$ at $x$ and $u$ is $\frac{2}{2} C_{x, u}(-,-)$. The two terms in (5.3) involving $D_{1} A$ will be shown to be $\bar{d} \bar{\omega}_{x}\left(w_{1}, w_{2}\right)(u)$, and the two terms involving $D_{2} A$ will be shown to be $\left[\bar{\omega}_{x}\left(w_{1}\right), \bar{w}_{x}\left(w_{2}\right)\right](u)$. Now the first is rather clear, since the vector space structure on $\operatorname{Vect}(F)$ is calculated in $T_{u} F$ for each fixed $u \in F$ separately. Keeping $u$ fixed and omitting it from notation, the expression

$$
D_{1} A\left(x, w_{2}\right)\left(w_{1}\right)-D_{1} A\left(x, w_{1}\right)\left(w_{2}\right)
$$

is the standard expression for the exterior differential of the 1-form given by $A$.

We now turn to calculating the tangent vector at $u$ involving the Lie bracket. Let us, for $\left(d_{1}, d_{2}\right) \in D \times D$ arbitrary, calculate

$$
\left[\bar{w}_{x}\left(w_{1}\right), \bar{w}_{x}\left(w_{2}\right)\right](u)\left(d_{1} \cdot d_{2}\right) \text {. }
$$




$$
\begin{aligned}
\bar{w}_{x}\left(w_{1}\right)\left(u, d_{1}\right) & =u+A\left(x, u, d_{1} \cdot w_{1}\right)=u^{\prime}, \\
\bar{w}_{x}\left(w_{2}\right)\left(u^{\prime}, d_{2}\right) & =u^{\prime}+A\left(x, u^{\prime}, d_{2} \cdot w_{2}\right) \\
& =u+A\left(x, u, d_{1} \cdot w_{1}\right)+A\left(x, u+A\left(x, u, d_{1} \cdot w_{1}\right), d_{2} \cdot w_{2}\right) \\
& =u+A\left(x, u, d_{1} \cdot w_{1}\right)+A\left(x, u, d_{2} \cdot w_{2}\right) \\
& +D_{2} A\left(x, u, d_{2} \cdot w_{2}\right)\left(A\left(x, u, d_{1} \cdot w_{1}\right)\right) .
\end{aligned}
$$

If instead we calculate

$$
\bar{\omega}_{x}\left(w_{1}\right)\left(\bar{\omega}_{x}\left(w_{2}\right)\left(u, d_{2}\right), d_{1}\right),
$$

and subtract, we are left with

$D_{2} A\left(x, u, d_{2} \cdot w_{2}\right)\left(A\left(x, u, d_{1} \cdot w_{1}\right)\right)-D_{2} A\left(x, u, d_{1} \cdot w_{1}\right)\left(A\left(x, u, d_{2} \cdot w_{2}\right)\right)$, and using linearity of $A$ in the third variable, this is

(5.4) $d_{1} \cdot d_{2} \cdot\left\{D_{2} A\left(x, u, w_{2}\right)\left(A\left(x, u, w_{1}\right)\right)-D_{2} A\left(x, u, w_{1}\right)\left(A\left(x, u, w_{2}\right)\right)\right\}$

proving that the field vector of the vector field

$$
\left[\bar{\omega}_{x}\left(w_{1}\right), \bar{\omega}_{x}\left(w_{2}\right)\right]
$$

at $u$ is the expression in the curly bracket in (5.4), which is the second line in (5.3), as desired. This proves the theorem.

The linear Maurer-Cartan form $\bar{\Omega}$ on a group $G$ is given by $t \mapsto\left[d \mapsto t(d) \cdot t(0)^{-1}\right]$, where $t: D \rightarrow G$ is an arbitrary tangent vector. If $G$ is a formal manifold, the $G$-valued Maurer-Cartan form $\Omega$ introduced in $\$ 2$,

$$
\Omega(x, y)=y \cdot x^{-1} \text { for } x \sim y
$$

corresponds to $\bar{\Omega}$. Since $\Omega$ is closed, (3.1) (being the coboundary of the identity map), we get, by setting the left hand side of (5.2) equal to 0 :

COROLLARY 5.5. The Zinear Maurer..Cartan form $\bar{\Omega}$ satisfies

$$
\partial \bar{\Omega}=-\frac{1}{2}[\bar{\Omega}, \bar{\Omega}] \text {. }
$$

This is the classical Maurer-Cartan formula. 


\section{Finite curve integrals}

As in [12], we shall assume that the basic ring $R$ (the line) is equipped with a preorder relation, making all intervals $[a, b]$ étale. We shall generalize the integration axiom for maps $[a, b] \rightarrow R$ : we say that the group $G$ admits integration if the following statement holds (that is, is internally valid):

(6.1) for all $a \leq b$ and for every $G$-valued 1 -form $\omega$ on the interval $[a, b]$ there exists a unique $g:[a, b] \rightarrow G$ with $g(a)=e$ and $d g=\omega$.

This generalizes the integration axiom [12] when one observes that an $(R,+)$-valued 1 -form on $[a, b]$ is of the form $f(x) d x$ for a unique $f:[a, b] \rightarrow R$. The proof of [12] that the "first" full Dubuc model [1] satisfies the integration axiom can be generalized to prove that any Lie group admits integration, but it does not apply to $\operatorname{Diff}(F)$. The classical fact used about Lie groups is the following:

PROPOSITION 6.1. Let (classically) $x_{t}$ be an ]a, b[-parametrized family of right invariant vector fields on a lie group $G$. Then there is a curve $g:] a, b[\rightarrow G$ with (for $a$ Iz $t \in] a, b[$ )

$$
\left.g^{\prime}(t)=X_{t}(g(t)) \text { for azz } t \in\right] a, b[\text {, }
$$

and any two such curves differ by right multiplication by a unique $\gamma \in G$.

Proof. For a $t$-parametrized vector field $X$ on a manifold $G$, we may to each $c \in] a, b[$ and each $\gamma \in G$ find an open interval around $c$ and a function $g$ defined on it with $g(c)=\gamma$ and satisfying (6.2). However, since our vector field $X_{t}$ (for each $t$ ) is right invariant, the interval around $c$ can be chosen uniformly, independent of the initial value $\gamma$. From this it is easy to prove that one can piece the local solutions together to a global one, $] a, b[\rightarrow G$.

Note that (6.1) implies that we can find a $g:[a, b] \rightarrow G$,

$$
g(y) \cdot g(x)^{-1}=\omega(x, y) \text { for all } x \sim y \text {, }
$$

or equivalently,

$$
g(y)=\omega(x, y) \cdot g(x) \text { for all } x \sim y .
$$


Such a $g$ we call a primitive or an indefinite (right) integral of $\omega$. (There is also a dual notion, asking for a $g$ satisfying $g(y)=g(x) \cdot \omega(x, y)$, but we shall not deal with it.) If $g$ is a primitive of $\omega$, then so is $g \cdot c$ for any $c \in G$, clearly.

Conversely, we may note that if $g$ and $\tilde{g}$ are two primitives of the 1 -form $w$ on $[a, b]$, then the uniqueness assertion in (6.1), applied to $g \cdot g(a)^{-1}$ and $\tilde{g} \cdot \tilde{g}(a)^{-1}$ allows us to conclude

$$
\tilde{g} \equiv g \cdot g(a)^{-1} \cdot \tilde{g}(a)
$$

so any two primitives of $\omega$ differ by right multiplication by a constant in $G$.

In the rest of $\S 6, G$ denotes a group admitting integration.

If $\omega$ is a $G$-valued $l$-form on $M$ (a formal manifold), and $k:[a, b] \rightarrow M$ is a curve on $M$, we define

$$
\int_{k} w:=g(b) \cdot g(a)^{-1}
$$

where $g:[a, b] \rightarrow G$ is a primitive of $k^{*} w$. Clearly, it does not matter which primitive we choose. Also, for $a \leq b \leq c$, we have, for any $k:[a, c] \rightarrow M$,

$$
\int_{k} \omega=\int_{k \mid[b, c]} \omega \cdot \int_{k \mid[a, b]} \omega
$$

and

$$
\int_{k \mid[a, b]} \omega=\omega(k(a), k(b)) \text { if } a \sim b .
$$

(Note that, like in [12], we implicitly retain the information of the end points in the notation $[a, b]$ : the interval is not determined by its underlying set.)

We can also, like in [12], prove that if

$$
f:\left[a_{1}, b_{1}\right] \rightarrow[a, b]
$$

has $f\left(a_{1}\right)=a, f\left(b_{1}\right)=b$, then for $k:[a, b] \rightarrow M$ we have 


$$
\int_{k} \omega=\int_{k \circ f} \omega,
$$

so that the integral of $\omega$ over a curve "does not depend on its parametrization" if we retain the information of its end points. This is the justification for the pictorial shorthand used in the following lemma; "I" denotes $[0,1]$.

LEMMA 6.2. Assume $\omega$ is a G-valued 1 -form on $I \times I$. Then the integrals of $\omega$ around the two paths from $(0,0)$ to $(1,1)$ are equal, provided the two integrals of $\omega$ around any infinitesimal rectangle $\left|a_{1}, a_{1}+d_{1}\right| \times\left|a_{2}, a_{2}+d_{2}\right|$ are equal.

Proof. This is much similar to the corresponding fact about $R$-valued integrals, as exposed in [13], or [11], §I.15. Consider the picture

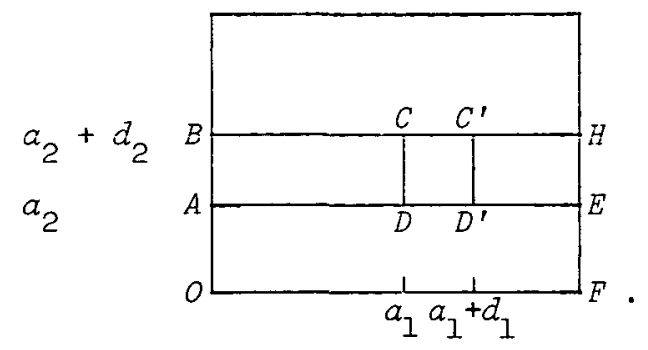

We first prove that the integral both ways round from $A$ to $H$ agree, by proving that the two functions $f_{i}: I \rightarrow G$ sending $a_{1}$ to, respectively,

$$
\int_{A B C} \omega \text { and } \int_{A D C} \omega
$$

have the same differential: $d f_{1}=d f_{2}$ (and, evidently, the same value for $a_{1}=0$, namely $\left.w\left(a_{2}, a_{2}+a_{2}\right)\right)$. This will imply, by the uniqueness assertion in the integration axiom, that $f_{1}=f_{2}$, hence $f_{1}(1)=f_{2}(1)$, which are the two desired integrals. We have 


$$
\begin{aligned}
d f_{1}\left(a_{1}, a_{1}+d_{1}\right) & =\int_{A D^{\prime} C^{\prime}} \cdot \int_{A D C}^{-1} \\
& =\int_{D^{\prime} C^{\prime}} \cdot \int_{D D^{\prime}} \cdot \int_{A D} \cdot \int_{A D}^{-1} \cdot \int_{D C}^{-1} \\
& =\int_{D^{\prime} C^{\prime}} \cdot \int_{D D^{\prime}} \cdot \int_{D C}^{-1}
\end{aligned}
$$

whereas

$$
\begin{aligned}
d f_{2}\left(a_{1}, a_{1}+d_{1}\right) & =\int_{C C^{\prime}} \cdot \int_{B C} \cdot \int_{A B} \cdot\left(\int_{B C} \cdot \int_{A B}\right)^{-1} \\
& =\int_{C C^{\prime}} \cdot
\end{aligned}
$$

However, the assumption about infinitesimal rectangles implies

$$
\int_{D^{\prime} C^{\prime}} \cdot \int_{D D^{\prime}}=\int_{C C^{\prime}} \cdot \int_{D C}
$$

from which the equality of (6.3) and (6.4) follows by multiplying by $\int_{D C}^{-1}$ on the right.

Next, we prove that the two functions $g_{i}: I \rightarrow G$, sending $a_{2}$ to, respectively

$$
\int_{O A E} \omega \text { and } \int_{O F E} \omega
$$

are equal, by proving that they have the same differential: $d g_{1}=d g_{2}$ (and evidently same value for $a_{2}=0$, namely $\int_{O F} \omega$ ). This proof is similar to the proof already carried out for the strip, and utilizing the result for the strip.

\section{When closed forms are exact}

Our main result will be on pathwise connected and simply connected formal manifolds. We need however for technical reasons a notion slightly stronger than "pathwise connected" namely "stably pathwise connected". A 
formal manifold $M$ of dimension $n$ will be said to have this property if it is pathwise connected (it is non-empty, and for any two points in it, there is a path $k: I \rightarrow M$ having the two given points as endpoints), and if it satisfies the conclusion of the following proposition.

PROPOSITION 7.1. Let $M$ be an etale subset of $R^{n}$. Then, for any path $k_{0}: I \rightarrow M$ and any $z \sim k_{0}(1)$, there is a sequence $k_{0}, \ldots, k_{n}$ of paths, all beginning in $k_{0}(0)=x$, and with $k_{n}(1)=z$, as well as an element $\left(d_{1}, \ldots, d_{n}\right) \in D(n)$, such that each path $k_{i}$ is homotopic to the path $k_{i+1}$ by a homotopy of duration $d_{i}$ keeping $x$ fixed.

(A 'homotopy of duration $d \in D$ ' is a map $I \times D \rightarrow M$, having the two desired paths as its restrictions on $I \times\{0\}$ and $I \times\{d\}$, respectively.)

Proof. Let $y=k_{0}(1), z=y+\mathrm{d}$ with $\mathrm{d}=\left(d_{1}, \ldots, d_{n}\right)$. Then we will construct the $k_{i}$ 's to have endpoints

$$
z+\left(d_{1}, \ldots, d_{i}, 0, \ldots, 0\right) \text {. }
$$

We define

$$
k_{i+1}(s, \delta)=k_{i}(s)+s \cdot(0, \ldots, \delta, \ldots, 0)
$$

with $\delta$ in the $(i+1)$ st position. The $n$ durations are taken to be $d_{1}, \ldots, d_{n}$, respectively.

To say that $M$ is simply connected, we mean that any two paths $k_{0}$ and $k_{1}$, beginning at $x$ and ending at $y$, can be connected by a homotopy $K: I \times I \rightarrow M$ keeping $x$ and $y$ fixed:

$$
\begin{aligned}
& K(t, i)=k_{i}(t), i=0,1, \text { for all } t \in I, \\
& K(0, s)=x \text { for all } s \in I, \\
& K(1, s)=y \text { for all } s \in I .
\end{aligned}
$$

We assume that the group $G$ admits integration, as in $\$ 6$.

THEOREM 7.2. If $M$ is a stably pathwise connected and simply connected formal manifold, then any closed G-valued 1 -form is exact. 
Proof. Let $\Theta$ be a closed 1-form, $d \theta=0$. Choose a point $x \in M$ ( $M$ is inhabited), and define, for $y \in M$,

$$
f(y)=\int_{k(x, y)} \Theta
$$

where $k(x, y)$ is any path $I \rightarrow M$ beginning in $x$ and ending in $y$. This is independent of the choice of path, for, if $k_{0}$ and $k_{1}$ are two such, we connect them with a homotopy $K$ as above. Then $\omega:=K^{*} \theta$ is a closed 1 -form on $I \times I$. This means that the curve integral (4.2) around infinitesimal triangles in $I \times I$ vanishes. By Proposition 4.1, therefore, the curve integral of $\omega$ around infinitesimal parallelograms vanishes also, or, equivalently, the curve integral both ways round from the lower left vertex to the upper right vertex in such a parallelogram are equal. By Lenma 6.2 the two curve integrals of $\omega$ around $I \times I$ are equal. But $\omega=K^{*} \Theta$ is the zero form on two of the sides of $I \times I$, because $K$ is constant here, and equals $k_{0}^{*} \Theta$ and $k_{1}^{*} \Theta$, respectively, on the other two. This means $\int_{k_{0}} \Theta=\int_{k_{1}} \Theta$, which is the desired conclusion for well-definedness of $f$.

We shall finally prove $d f=\theta$. Let $y \sim z$, and choose a path $k_{0}$ from $x$ to $y$, as well as paths $k_{1}, \ldots, k_{n}$ with $k_{n}(1)=z$, as in the conclusion of Proposition 7.1, using $M$ stably pathwise connected. Mapping $I$ in an affine way to $\left[0, d_{i}\right]$ by mapping 0 to 0 and $I$ to $d_{i}$ reparametrizes the $i$ th infinitesimal homotopy in Proposition 7.1 to an I-parametrized one, and Lemma 6.2 applies again. This time, the 1-end of the homotopy gives a contribution, namely, as is easily seen,

$$
\theta\left(k_{i}(1), k_{i+1}(1)\right) \text {. }
$$

The 'difference' between $\int_{k_{0}} \theta$ and $\int_{k_{n}} \theta$, that is $f(z) \cdot f(y)^{-1}$, is therefore

$$
\theta\left(k_{n-1}(1), k_{n}(1)\right) \cdot \ldots \cdot \theta\left(k_{0}(1), k_{1}(1)\right)
$$

Since $\theta$ is closed, and $k_{i-1}(1), k_{i}(1), k_{i+1}(1)$ form an infinitesimal 
triangle,

$$
\theta\left(k_{i}(1), k_{i+1}(1)\right) \cdot \theta\left(k_{i-1}(1), k_{i}(1)\right)=\theta\left(k_{i-1}(1), k_{i+1}(1)\right)
$$

so the expression (7.1) reduces to

$$
\Theta\left(k_{0}(1), k_{n}(1)\right)=\Theta(y, z) .
$$

This proves $d f=\theta$, and thus the theorem.

We may read Theorem 7.2 as giving conditions when a formal manifold $M$ has the property that closed I-forms are exact, or $H^{1}(M, G)=0$ (deRham cohomology with values in $G$ ). The next theorem similarly deals with O-forms, or $H^{0}(M, G)$.

THEOREM 7.3. If $M$ is a pathwise connected inhabited (that is, 'non-empty') formal manifold, then any map $h: M \rightarrow G$ with $d h=0$ is constant.

Proof. Choose an $x \in M$ ( $M$ being inhabited). We prove $h(y)=h(x)$ for all $y \in M$. By pathwise connectedness, we may find a path $k:[a, b] \rightarrow M$ with $k(a)=x, k(b)=y$. It suffices to see that $h \circ k$ is constant. Now

$$
d(h \circ k)=k^{*}(d h)=k *(0)=0 .
$$

So $h \circ k$ is a primitive of the zero 1 -form on $[a, b]$; clearly the map $\bar{e}$ with constant value $e$ is also such. Since any two primitives of a I-form differ by right multiplication by a constant, we conclude

$$
h \circ k=\bar{e} \cdot g
$$

or $h \circ k \equiv g$.

Theorems 7.2 and 7.3 are equivalent to the 'Two lemmas on MaurerCartan forms' from, say, [4]; cf. also [6].

COROLLARY 7.4. Let $M$ and $G$ be as in Theorem 7.3, and let $f, \tilde{f}: M \rightarrow G$ be maps. Then

$$
f(x)=\tilde{f}(x) \cdot g
$$

for fixed $g \in G$ if and only if

$$
f^{*} \Omega=\tilde{f} * \Omega
$$

where $\Omega$ is the Maurer-Cartan form on $G$; equivalently, if and only if 


$$
d f=\tilde{f} .
$$

Proof. The equivalence of the last two conditions is immediate from (3.2) $\left(d f=f^{*} \Omega\right)$. If $f(x)=\tilde{f}(x) \cdot g$, one immediately gets $d f=d \tilde{f}$, so assume finally the latter. Consider

$$
g(x):=\tilde{f}(x)^{-1} \cdot f(x)
$$

and calculate, for $x \sim y$,

$$
\begin{aligned}
d g(x, y) & \left.=\tilde{f}(y)^{-1} f(y) \cdot \tilde{f}(x)^{-1} \cdot f(x)\right)^{-1} \\
& =\tilde{f}(y)^{-1} \cdot f(y) \cdot f(x)^{-1} \cdot \tilde{f}(x) \\
& =\tilde{f}(y)^{-1} \cdot d f(x, y) \cdot \tilde{f}(x) \\
& =\tilde{f}(y)^{-1} \cdot \tilde{f f}(x, y) \cdot \tilde{f}(x) \\
& =e,
\end{aligned}
$$

so by Theorem $7.3, g$ is constant.

Clearly, (7.3) holds for the non-Iinear (G-valued) Maurer-Cartan form if and only if it holds for the linear ( $L G$-valued) one. When we read (7.3) the latter way, the equivalence $(7.2) \Leftrightarrow(7.3)$ is Lemma 1.3 in [4].

COROLLARY 7.5. Let $M$ and $G$ be as in Theorem 7.2, and let $\bar{\varphi}$ be a linear 1-form on $M$ with values in $L G$. Then the following two conditions are equivalent:

(7.5) there exists a map $f: M \rightarrow G$ with $f * \bar{\Omega}=\bar{\varphi}$ (where $\bar{\Omega}$ is the Zinear Maurer-Cartan form);

$$
\bar{d} \bar{\varphi}=-\frac{3}{2}[\bar{\varphi}, \bar{\varphi}] \text {. }
$$

Proof. If (7.5) holds, (7.6) follows from the Maurer-Cartan formula (Corollary 5.5). Conversely, assume (7.6). Let $\varphi$ be the (non-linear) 1-form on $M$ with values in $G$ corresponding to $\bar{\varphi}$. Then, by (5.2),

$$
\overline{d \varphi}=\frac{2}{2}\left(\bar{d} \bar{\varphi}+\frac{1}{2}[\bar{\varphi}, \bar{\varphi}]\right),
$$

which is zero, by $(7.6)$, so $d \varphi=0$. Hence by Theorem $7.2, \varphi$ is exact:

$$
\varphi=d f
$$

for some $f: M \rightarrow G$, or, equivalently by (3.2),

$$
\varphi=f^{*} \Omega,
$$

whence 


$$
\bar{\varphi}=\overline{f^{*} \Omega}=f^{\star} \bar{\Omega} .
$$

This proves the corollary.

The corollary is Lemma (1.4) in [4], except for a minus sign, due to different conventions.

\section{Remarks on Frobenius distributions}

We consider a distribution $D$ on $M \times F$, transversal to the fibres of the projection $P: M \times F \rightarrow M$, as in $\S 1$, with $M$ and $F$ formal manifolds. As there, we get a $\operatorname{Diff}(F)$-valued 1 -form $\omega$ on $M$ ('the connection form'). It is characterised by the fact that, for all $x \sim y$ in $M$, and $u, u^{\prime} \in F$,

$$
w(x, y)(u)=u^{\prime} \text { if and only if }\left(y, u^{\prime}\right) \in \mathcal{D}(x, u) \text {. }
$$

We say that a tangent $t: D \rightarrow M \times F$ is subordinate to $D$ if $t(d) \in D(t(0))$ for all $d \in D$. For technical reasons, we need the following condition on $D$ :

(8.2) for a $t: D \rightarrow M \times F$ to be subordinate to $D$, it suffices that $t\left(d_{1} \cdot d_{2}\right) \in D(t(0))$ for all $\left(d_{1}, d_{2}\right) \in D \times D$.

A vector field $X$ on an étale subset $U$ of $M \times F$ is called subordinate to $D$ if each of its field vectors are, that is, if $X(s, d) \in D(s)$ for all $s \in U$ and $d$. We say that $D$ is a Frobenius distribution if the Lie bracket $[X, Y]$ of any two $D$-subordinate vector fields $X$ and $Y$ is again $D_{\text {-subordinate. }}$

This aim of this section is to give a geometric proof (a proof not involving any calculations with + , only composition in the group $\operatorname{Diff}(F)$ ) of (1) $\Leftrightarrow(2)$ in the following theorem (where (1) $\Longleftrightarrow(3)$ is classical; see, for example, [5]).

THEOREM 8.1. Let $D$ be a distribution on $M \times F$, transversal to the fibres of the projection to $M$, and assume (8.2) holds. Then the following three statements are equivalent:

(1) $D$ is a Frobenius distribution;

(2) $d \omega=0$ (where $\omega$ is the Diff(F)-valued connection form);

(3) $d \bar{\omega}=-\frac{1}{2}[\bar{\omega}, \bar{\omega}]$ (where $\bar{\omega}$ is the Vect $(E)$-valued linearized 
connection form).

Proof. From Theorem 5.4 follows the equivalence of (2) and (3). To prove $(1) \Leftrightarrow(2)$, we need a lemma, concerning some further "infinitesimal curve integrals", amending Proposition 4.1. Let $X$ and $Y$ be vector fields on a formal manifold $N$, and consider, for some fixed $m \in N$ and $\left(d_{1}, d_{2}\right) \in D \times D$ the five points (cf., for example, [11], I.9.3)

$$
m, n=X\left(m, d_{1}\right), p=Y\left(n, d_{2}\right), q=X\left(p,-d_{1}\right), r=Y\left(q,-d_{2}\right)
$$

so that $r=[X, Y]\left(m, d_{1} \cdot d_{2}\right)$. (These five points form what we may call a Lie-pentagon for $\left.x, y, d_{1}, d_{2}.\right)$

LEMMA 8.2. For any closed G-valued 1-form $\theta$, the "curve integral" of $\theta$ around the pentagon $m, n, p, q, r$, is $e$ :

$$
\theta(r, m) \cdot \theta(q, r) \cdot \theta(p, q) \cdot \theta(n, p) \cdot \theta(m, n)=e .
$$

Proof. Since everything is local, we may assume that $N$ is an étale subset of $R^{n}$. The proof now consists in dissecting the pentagon into an infinitesimal parallelogram as in (4.5), and three infinitesimal triangles as in (4.4). Around the parallelogram and around each of the triangles, the 'curve-integral' in question is 0 , by $d \theta=0$ and Proposition 4.1. The dissection is constructed as follows. We may assume

$$
X(x, d)=x+d \cdot \xi(x) \text { for all } x \in N \text { and } d \in D,
$$

for some $\xi: N \rightarrow R^{n}$ ("the principal part of $X "$ ); this follows from the basic axiom for $R$. Similarly, $Y$ has a principal part $\eta: N \rightarrow R^{n}$. The five points under consideration are then

$$
\begin{aligned}
m & , \\
n & =m+d_{1} \cdot \xi(m), \\
p & =m+d_{1} \cdot \xi(m)+d_{2} \cdot n\left(m+d_{1} \cdot \xi(m)\right) \\
& =m+d_{1} \cdot \xi(m)+d_{2} \cdot n(m)+d_{1} \cdot d_{2} \cdot X(n)(m)
\end{aligned}
$$

(where $X(n)$ is the partial derivative of $n$ in the direction of the vector field $X$ ); and similarly

$$
q=m+d_{2} \cdot \eta(m)+d_{1} \cdot d_{2} \cdot(X(n)(m)-Y(\xi)(m)),
$$




$$
x=m+d_{1} \cdot d_{2} \cdot(X(n)(m)-Y(\xi)(m)) .
$$

Then

$$
\begin{aligned}
& m=m, \\
& n=m+d_{1}, \\
& p=m+d_{1}+d_{2}+\delta_{1}, \\
& q=m \quad+d_{2}+\delta_{1}-\delta_{2}, \\
& r=m \quad+\delta_{1}-\delta_{2},
\end{aligned}
$$

where $d_{1}=d_{1} \cdot \xi(m), d_{2}=d_{2} \cdot n(m), \delta_{1}=d_{1} \cdot d_{2} \cdot X(n)(m)$, and $\delta_{2}=d_{1} \cdot d_{2} \cdot Y(\xi)(m)$. We further consider

$$
q^{\prime}:=m+\mathrm{d}_{2}+\delta_{1} \text {. }
$$

Then the parallelogram 'mpq' is of the kind considered in (4.5) (note $d_{2}+\delta_{1} \in D(n)$ because $d_{2}$ and $\delta_{1}$ both contain $d_{2} \in D$ as a factor), and we have the triangles $p q q^{\prime}, q q^{\prime} m$, $q \mathbf{r}^{\prime}$, each of which are of the kind considered in (4.4). Note, for instance, that

$$
\begin{aligned}
q & =p-d_{1}-\delta_{2}, \\
q^{\prime} & =p-d_{1}
\end{aligned}
$$

and $\left(-d_{1}-\delta_{2},-d_{1}\right) \in \tilde{D}(2 n)$ because both $d_{1}$ and $\delta_{2}$ contain the factor $d_{1} \in D$. This proves the lemma.

To prove $(2) \Rightarrow(1)$ in the theorem, let $S$ and $T$ be $D$-subordinate vector fields on an étale subset $U \subseteq M \times F$, and consider a Lie pentagon for $S, T, d_{1}, d_{2}: \mathrm{m}=\left(m, v_{1}\right), \mathrm{n}=\left(n, v_{2}\right), \ldots, r=\left(r, v_{5}\right)$, say.

Because the vector fields $S$ and $T$ are $D$-subordinate, we get from (8.1) the second equality sign in

$$
\left(p^{*} \omega\right)\left(\left(m, v_{1}\right),\left(n, v_{2}\right)\right)\left(v_{1}\right)=w(m, n)\left(v_{1}\right)=v_{2} ;
$$

similarly

$$
\omega(n, p)\left(v_{2}\right)=v_{3}, \omega(p, q)\left(v_{3}\right)=v_{4}, \omega(q, r)\left(v_{4}\right)=v_{5},
$$


so that the "curve integral" of $p^{*} \omega$ around the first four legs of the pentagon is an element of $\operatorname{Diff}(F)$ which sends $v_{1}$ to $v_{5}$. But because $p^{*} \omega$ is closed, it follows from the lemma that the "curve integral" of $p^{*} \omega$ around the whole pentagon is zero (equals id $\in \operatorname{Diff}(F)$ ), so that we must have

$$
p^{*}(\omega)\left(\left(r, v_{5}\right),\left(m, v_{1}\right)\right)\left(v_{5}\right)=v_{1}
$$

or equivalently

$$
\omega(m, r)\left(v_{1}\right)=v_{5}
$$

or equivalently, by $(8.1),\left(r, v_{5}\right) \in O\left(m, v_{1}\right)$. But

$$
\left(r, v_{5}\right)=[S, T]\left(\left(m, v_{1}\right), d_{1} \cdot d_{2}\right),
$$

by the definition of Lie brackets in terms of Lie pentagons. Assumption (8.2) then allows us to conclude that the tangent $[S, T]\left(\left(m, v_{1}\right),-\right)$ is subordinate to $D$. Since $\left(m, v_{1}\right)$ was an arbitrary element in $U \subseteq M \times F$, we conclude that $[S, T]$ is 0 -subordinate.

Finally, to prove ( $]) \Rightarrow(2)$, assume that $D$ is a Frobenius distribution. To prove $d \omega=0$, it suffices to consider the restriction of $\omega$ to an arbitrary $\infty_{-m o n a d}$ in $M$, which we in turn may identify with $D_{\infty}(n) \subseteq R^{n}$; in other words, let us work in coordinates. By Proposition 4.I, it suffices to prove that the "curve integral" (4.5) around an infinitesimal parallelogram is zero. Consider, for fixed $m \in D_{\infty}(n) \subseteq R^{n}$ the value $(4.5)$ as a function of $(d, \delta)$, so as a function $D(n) \times D(n) \rightarrow G=\operatorname{Diff}(E)$. To prove that it is constant with value $e \in G$, it suffices, by infinitesimal linearity of $G$, to prove that its value is $e$ on elements in $D(n) \times D(n)$ of form $\left(d_{1} \cdot \mathrm{e}_{i}, d_{2} \cdot \mathrm{e}_{j}\right)$, where $\mathrm{e}_{i}$ and $\mathrm{e}_{j}$ are any two of the canonical basis vectors of $R^{n}$. Keeping $i$ and $j$ fixed, we have two vector fields $X$ and $Y$ on $D_{\infty}(n)$, with principal part constant equal to $\mathrm{e}_{i}$ and $\mathrm{e}_{j}$, respectively. Clearly the vector fields commute: $[X, Y]=0$. Before proceeding, we need some generalities about lifts of vector fields.

For any tangent vector $t: D \rightarrow M$ and $u \in F$, there is a unique 
$t: D \rightarrow M \times F$ satisfying

$$
\tilde{t}(0)=(t(0), u) \text { and } \tilde{t} \text { subordinate to } 0 \text {, }
$$

which we call the lift of $t$ starting in $u$ (relative to 0 ). We clearly have

$$
t(d)=(t(d), w(t(0), t(d))(u))
$$

where $\omega$ is the connection form for $D$.

For a vector field $Z$ on $U \subseteq M$, there is a lifted vector field $\tilde{Z}$ on $U \times F$, namely

$$
\tilde{Z}(x, u)=\text { lift of } Z(x) \text { starting in } u \text {, }
$$

and $\tilde{Z}$ is a D-subordinate vector field. For an arbitrary distribution $D,\left[z_{1}, z_{2}\right]^{\sim}$ is in general different from $\left[\tilde{z}_{1}, \tilde{z}_{2}\right]$, but if $D$ is a Frobenius distribution,

$$
\left[z_{1}, z_{2}\right]^{\sim}=\left[\tilde{z}_{1}, \tilde{z}_{2}\right]
$$

For, by the Frobenius property, both are D-subordinate, and both project to $\left[Z_{1}, Z_{2}\right]$ by $p$ (meaning that the field vector of either of them at any point of the fibre over $m$ projects to the field vector of $\left[z_{1}, z_{2}\right]$ at $m$ ). However, by uniqueness of lifts of tangent vectors, there is at most one such vector field.

Returning to the proof, since $D$ is Frobenius and $X$ and $Y$ commute, we have by $(8.4)$ that $[\tilde{X}, \tilde{Y}]=0$, that is, the lifts $\tilde{X}$ and $\tilde{Y}$ commute, which means that in any Lie pentagon for $\tilde{X}$ and $\tilde{Y}$, the first and fifth points are equal. So, for $m \in D_{\infty}(n), v_{1} \in F$, $\left(d_{1}, d_{2}\right) \in D \times D$, we have

$$
\tilde{Y}\left(\tilde{X}\left(\tilde{Y}\left(\tilde{X}\left(\left(m, v_{1}\right), d_{1}\right), d_{2}\right),-d_{1}\right),-d_{2}\right)=\left(m, v_{1}\right) .
$$

Using (8.3), we can rewrite this in terms of the connection form; by $(8.3)$,

$$
\begin{aligned}
\tilde{X}\left(\left(m, v_{1}\right), d_{1}\right) & =\left(m, \omega\left(m, x\left(m, d_{1}\right)\right)\left(v_{1}\right)\right) \\
& =\left(m, \omega\left(m, m+d_{1} \cdot \mathrm{e}_{i}\right)\left(v_{1}\right)\right)
\end{aligned}
$$

substituting into ( 8.5 ) and doing a similar thing three more times, we 
finally calculate that the left hand side of (8.5) appears as $\left(r, v_{5}\right)$ with $v_{5}$ equal to the result of applying the element of $\operatorname{Diff}(F)$ displayed in (4.5) to $v_{1}$ (provided we in (4.5) write $a_{1} \cdot e_{i}$ for $d$, $d_{2} \cdot \mathrm{e}_{j}$ for $\delta$, and $m$ for $x$ ). Since, by $(8.5), v_{5}$ also equals $v_{1}$, we conclude that the element (4.5) has trivial action on $v_{1}$. Since $v_{1}$ was arbitrary, this proves (4.5) equal to $e \in \operatorname{Diff}(F)$. As argued, this implies $d w=0$. This finishes the proof of the theorem.

We note that $\operatorname{Diff}(F)$ is "too big" to admit integration, in general. in other words, the three equivalent conditions on $\omega$ in the theorem do not imply that $\omega=d f$ for some $f: M \rightarrow \operatorname{Diff}(F)$. One would not expect that: the Frobenius distribution on $R \times R$ given by the differential equation $y^{\prime}=y^{2}$ has the property that for no proper interval on the $x$-axis (around $x_{0}$, say) does there, for every initial value $\left(x_{0}, y\right)$, exist a solution extending over the interval.

I still do not know how to formulate, in a way adequate for the synthetic setting, the Zocal integration which $\operatorname{Diff}(F)$ should have. One would probably have to replace the "global" group Diff(F) by the "inductive groupoid" [3], or "pseudogroup" of local diffeomorphisms of $F$.

\section{References}

[1] Eduardo J. Dubuc, "Sur les modèles de la géométrie differentielle synthétique", Cahiers Topologie Géom. Différentielle 20 (1979), 231-279.

[2] Charles Ehresmann, "Les connexions infinitésimales dans un espace fibré différentiable", Colzoque de topologie (espaces fibrés), Bruxelles, 1950, 29-55 (Georges Thone, Liège; Masson, Paris; 1951).

[3] Charles Ehresmann, "Catégories inductives et pseudogroupes", Ann. Inst. Fourier (Grenoble) 10 (1960), 307-332. 
[4] P. Griffiths, "On Cartans' method of Lie groups and moving frames as applied to uniqueness and existence questions in differential geometry:", Duke Math. J. 41 (1974), 775-814.

[5] André Haefliger, "Cohomology of Lie algebras and foliations", Differential topology, foliations and Gelfand-Euks cohomology, 1-12 (Proc. Sympos. Pontifica Universidade Católica do Rio de Janeiro, 1976. Lecture Notes in Mathematics, 652. SpringerVerlag, Berlin, Heidelberg, New York, 1978).

[6] Robert Hermann, "On the differential geometry of foliations", Ann. of Math. 72 (1960), 445-457.

[7] Anders Kock, "Taylor series calculus for ring objects of line type", J. Pure Appl. Algebra 12 (1978), 271-293.

[8] Anders Kock, "On the synthetic theory of vector fields", Topos theoretic methods in geometry, 139-157 (Various Publications Series, 30. Matematisk Institut, Aarhus Universitet, Aarhus, 1979).

[9] Anders Kock, "Properties of well-adapted models for synthetic differential geometry", J. Pure App Z. Algebra 20 (1981), 55-70.

[10] Anders Kock, "Formal manifolds and synthetic theory of jet bundles", Cahiers Topologie Géom. Différentielze 21 (1980), 227-246.

[11] Anders Kock, Synthetic differential geometry (London Mathematical Society Lecture Notes, 51. Cambridge University Press, Cambridge, 1981).

[12] A. Kock and G.E. Reyes, "Models for synthetic integration theory", Math. Scand. 48 (1981), 145-152.

[13] A. Kock, G.E. Reyes, and B. Veit, Forms anä integration in syntnetic differential geometry (Aarhus Preprint Series, 31. Matematisk Institut, Aarhus Universitet, Aarhus, 1979/80).

[14] G.E. Reyes and G.C. Wraith, A note on tangent bundles in a category with a ring object", Math. Scand. 42 (1978), 53-63.

Matematisk Institut,

Aarhus Universitet,

Aarhus,

Denmark. 